\title{
A Functionalist Theory of Properties
}

\author{
ANN WHITTLE \\ University of Manchester
}

\begin{abstract}
I consider a grand, yet neglected proposal put forward by Shoemaker - a functionalist theory of all properties. I argue that two possible ways of developing this proposal meet with substantial objections. However, if we are prepared to endorse an ontology of tropes, one of these functionalist analyses can be developed into an original and informative theory of properties.
\end{abstract}

In his paper, 'Some Varieties of Functionalism,' Shoemaker suggests that 'the Ramsey-Lewis technique,' employed by functionalists in the philosophy of mind and beyond, can be extended to provide an analysis of all genuine properties, so that 'every property will be a functional property." Shoemaker's proposal is tantalizing. Does functionalism hold the key to understanding the nature of properties? By utilising functionalist techniques, can progress be made on the age-old question of what properties are?

Despite burgeoning interest in Shoemaker's causal theory of properties, of which this functionalist proposal is a form, these questions have received no direct attention. In this paper, I intend to fill this void, in the hope that a positive answer may be forthcoming. My strategy will be as follows: I shall begin by briefly outlining the Ramsey-Lewis technique of which Shoemaker speaks, after which I will look at two fundamentally different forms of functionalism in the philosophy of mind.

See Shoemaker (1981 p.275). There is a little bit of dramatic license here. Shoemaker thinks that the theory holds of all genuine properties, i.e., those whose 'acquisition or loss by a thing constitutes a genuine change in that thing' (see Shoemaker, 1980 p.207). It will become clear that this analysis couldn't apply to those properties without a functional role (e.g., plausibly, properties of abstract objects). But, at least at its very widest, it can be taken to be a theory of all those properties which have a functional role. From now on, this is what I shall mean by 'all properties.' 
Once this groundwork has been done, I shall consider what consequences follow from extending these two functional analyses to all properties. Although substantial problems will be encountered, I shall suggest that one of these analyses can be developed into an original and informative theory of properties.

\section{II}

Since Lewis, it has become standard practice for functionalists within the philosophy of mind to employ the 'Ramsey-Lewis technique' in order to explicate their theory. This offers a way of formulating a functional definition for mental predicates. But the technique is not exclusive to mental predicates, it can be employed whenever the entity specified by the predicate in question occupies a functional role. Although there are different ways of spelling out this technique, Lewis's formulation has become standard. We start by stating the theory for the predicate(s) in question - the T-term(s). This should say how the referent(s) of the predicate(s) causally interrelate with the referents of other, already understood, terms (the O-terms). Suppose, for instance, that the mental predicate 'is irritable' is defined by this very simple, toy theory:

(T) In all animals, hunger and tiredness cause irritability and irritability causes disproportionate behavioural responses to minor annoyances.

Following Block, let us write $(\mathrm{T})$ as $\mathrm{T}\left(\mathrm{S}_{1} \ldots \mathrm{S}_{\mathrm{n}}, \mathrm{I}_{1} \ldots \mathrm{I}_{\mathrm{n}}, \mathrm{O}_{1} \ldots \mathrm{O}_{\mathrm{n}}\right)$, where S's are the mental states, I's are the inputs and O's are the outputs. ${ }^{2}$

This theory, according to Lewis, provides us with an implicit definition of the predicate 'is irritable,' but by utilising the Ramsey-Lewis technique, we can turn it into an explicit definition of this term. First, we write the Ramsey sentence of the theory by replacing the T-terms with variables and prefixing existential quantifiers to the theory. In our theory, $F_{1}$ will replace the T-term 'is irritable,' so we say that there is a set of entities, $\exists F_{1 \ldots} \exists F_{n}$, that satisfies this formula: $T\left(F_{1} \ldots F_{n}, I_{1} \ldots I_{n}\right.$, $\left.\mathrm{O}_{1} \ldots \mathrm{O}_{\mathrm{n}}\right)$. Next, the Ramsey sentence is modified, to exclude the possibility of there being more than one state of which everything the theory says is true of it: $\exists F_{1 \ldots} \exists F_{n} T\left(F_{1} \ldots F_{n}, I_{1} \ldots I_{n}, O_{1} \ldots O_{n}\right) .^{3}$ Finally, we

See Block (1978).

The uniqueness constraint is required because if the T-terms name more than one thing, then they will name the components of some realisation or other. But, as Lewis notes, this 'does not tell us which one. So either the T-terms do not name anything, or they name the components of an arbitrarily chosen one of the realisations of T' (Lewis, 1970 p.432). This seems unacceptable. 
form the Carnap sentence of the theory by stating the conditional of the modified Ramsey sentence. So we say that if there is an $F_{1}$ such that everything the theory says is true of it, then that property of being $F_{1}$ will be the property of being irritable.

Shoemaker's innovative suggestion is that we make wholesale use of this Ramsey-Lewis technique. He writes,

If we could specify ail of the causal features of a property in a set of propositions of finite length, then using that set of propositions as our 'theory' we could use the Ramsey-Lewis technique to construct a functional predicate which is true of a thing...just in case it has that property. (1981, p.274)

To illustrate, suppose that the non-mental predicate 'is $100^{\circ} \mathrm{C}$ ' is defined by this very simple, toy theory:

(T) For all substances, if that substance is water and is heated to $100^{\circ} \mathrm{C}$, then this will cause that substance to boil and it will scald human skin on contact.

Once again, (T) can be written as $T\left(S_{1} \ldots S_{n}, I_{1} \ldots I_{n}, O_{1} \ldots O_{n}\right)$, where $S$ 's are various states, such as being water and being $100^{\circ} \mathrm{C}$, I's are the inputs, such as heating the water, and O's are the causal outputs, such as boiling and scalding human skin. This postulate can then be turned into a modified Ramsey sentence. So we say that there is a unique n-tuple of entities, $\exists F_{1 \ldots} \exists F_{n}$, which satisfies the formula $T\left(F_{1} \ldots F_{n}\right.$, $I_{1} \ldots I_{n}, O_{1} \ldots O_{n}$ ), and the variable $F_{1}$ replaces the $T$-term in question, namely 'is $100^{\circ} \mathrm{C}$.' Finally, we state the Carnap sentence for the theory by saying that if there is an $F_{1}$ such that everything the theory says is true of it, then that property of being $F_{1}$ is the property of being $100^{\circ} \mathrm{C}$.

The central issue that we shall be focusing upon here is what the resulting Ramsey-Lewis definitions of predicates tell us about the nature of their referents. But, before this, attention must first be drawn to a fundamental division within functionalist accounts. As the RamseyLewis technique outlined above follows the dictates of Lewis, it presents us with his form of functionalism, often referred to as functional realiser theory. ${ }^{4}$ What is distinctive about this view is that it takes the property of, say, being irritable, to be the property which causes the animal to overreact to minor annoyances, and whose instantiation is

4 The view is also known as the 'functional specification theory' (see, for instance, Block, 1980 p.179). 
caused by an animal's hunger and tiredness. In other words, it is the property which occupies the $F_{1}$ role in our Ramsey-Lewis definition.

The form of functionalism that originates from the work of Putnam, however, differs significantly from this. ${ }^{5}$ So called functional role theorists argue that functional properties are higher-order properties. ${ }^{6}$ They are properties which, in Block's words, 'consist in the having of some properties or other...that have certain causal relations to one another." Instead of taking the property of being irritable to be the property which stands in the $F_{1}$ role, functional role theorists claim that the property of being irritable is the property of possessing some property which occupies the $F_{1}$ role.

In the philosophy of mind, the claimed phenomenon of multiplerealisability moves functional role theorists to adopt this view. Functional role theorists believe that many different kinds of properties could and perhaps do occupy the $F_{1}$ role. Consequently, there is no unique property occupying $F_{1}$, with which the property of being irritable can be identified. This commitment is clearly at odds with the Ramsey-Lewis technique as outlined above, for this has a uniqueness constraint built into it. It seems unwise simply to jettison this constraint, since then it becomes extremely unclear what our T-term is supposed to refer to. However, we can regain the uniqueness of the definitions by utilising the following procedure: First, we restrict the Ramsey-Lewis definition of 'is irritable' to systems where there is one property for each predicate variable. This is to ensure that, within that particular domain, there is a unique physical property which always occupies the $F_{1}$ role. I shall call this unique matrix of properties a 'concrete realisation' of the mental theory for irritability. If functional role theorists are correct, there will be many different systems and so many different concrete realisations of this theory. So the property of being irritable cannot be identified with any one of the physical properties

5 See Putnam (1967). I shall refer to this form of functionalism as 'functional role theory', but it is also known as 'functional state identity theory' (see, for instance, Block, 1980 p.179).

6 Originally, talk of different orders of properties belonged to Russell's ramified theory of types. There, every type of entity was further split into orders of entity. Take, for instance, any type 1 property (i.e., those that apply significantly to individuals). We can split this type further into orders. The first-order of properties will be those properties of individuals expressed by predicates which either contain no quantifiers, or only quantifiers whose domain is individuals. The second-order will be all those properties expressed by predicates whose definitions require quantification over firstorder properties. The third-order will be all those properties expressed by predicates whose definitions require quantification over second-order properties, and so on.

See Block (1980, p.155). 
that occupies the $F_{1}$ role in a concrete realisation, since none of these properties are able to realise $F_{1}$ 's functional role in all systems.

In order to regain a unique property which can be identified with irritability, functional role theorists need to abstract away from the differences of these concrete realisations and focus only upon their shared aspects. In this way, they can isolate the functional role that is common to the distinct concrete realisations, and so identify the property of being irritable with this abstract functional role. But how can a new type of entity be abstracted from a set of physical properties, all of which occupy functional role $F_{1}$ in a particular system?

We can isolate the common feature of these concrete realisations by utilising a Fregean abstraction principle of the form $(\forall x)(\forall y)(f(x)=$ $\mathrm{f}(\mathrm{y}) \leftrightarrow \mathrm{x} \approx \mathrm{y}){ }^{8}$ Abstraction principles enable us to identify new types of entities as equivalence classes of an already familiar type. The identity conditions for these new entities are given by an equivalence relation defined on the original, familiar entities. For instance, by defining an equivalence relation that holds between lines, namely parallelism, Frege provides a necessary and sufficient condition for the identity of directions. The same move can be made in the present case. If the variables in the abstraction principle are taken to range over the familiar concrete realisations of the mental theory for irritability, the functional role common to all of them can be isolated by this abstraction principle: the functional role of concrete realisation $\mathrm{x}$ is identical to the functional role of concrete realisation $\mathrm{y}$ iff $\mathrm{x}$ exemplifies the form of the same mental theory as $y$.

Controversy rages over how these abstraction principles should be interpreted. Should functional roles be identified with the equivalence class of concrete realisations? Or, are there such things as functional roles that are independent of their concrete realisations, with which the property of being irritable can be identified?' ${ }^{9}$ Functional realisers in the philosophy of mind, such as Lewis, can be seen as opposing this latter claim, as they are sceptical of the idea that there are such things as functional roles, which are distinct from their physical concrete realisations. There aren't any properties of being such-and-such functional roles, there are only the properties which realise these functional roles. Functional role theorists, on the other hand, think that the functional role which is abstracted from the various concrete realisations of a mental theory is

8 This states that the function $\mathrm{f}$ of $\mathrm{x}$ is identical to the function $\mathrm{f}$ of $\mathrm{y}$ iff there is an equivalence relation between the entities over which variables $x$ and $y$ range. These abstraction principles are also referred to as 'second-order criterion of identity' (see Williamson, 1990 p.145).

9 Dummett (1991) puts forward the reductionist reading, while Wright (1983) defends the anti-reductionist view. 
something distinct from those concrete realisations. So the property of being irritable can be identified with this unique, abstract functional role.

The fundamental divide between these two forms of functionalism-functional realiser and functional role theory-raises a question: Should a wholesale functionalist theory of properties follow the dictates of Lewis or Putnam? I think that Shoemaker leaves the answer to this question somewhat unclear. In the subsequent two sections, I will argue that neither route appears promising. I shall begin by looking at the consequences of adopting a functional realiser theory of properties.

\section{III}

The process of extending functional realiser theory to all properties seems unproblematic. So long as the scope of the theory is limited, in the way suggested earlier, to those properties capable of making a contribution to the causal nexus of the world, there will be a possible theory stating what particulars are enabled to do in virtue of instantiating that property. ${ }^{10}$ Using the Ramsey-Lewis technique, this can then be turned into an explicit functional definition for that property's predicate. The question which requires consideration, then, is this: What does our comprehensive array of functional definitions tell us about the nature of the properties thus defined? In particular, does it enable us to make progress on the age-old question of what properties are?

It is difficult to see how such a theory does or could offer the illuminating analysis we are after. For these Ramsey-Lewis definitions aim at conceptual clarification. They tell us how we can define the T-terms via the already accepted O-terms. But they do not offer any metaphysical information about the referents of the T-terms. Consequently, no matter how wide the scope of functional realiser theory is taken to be, an interesting metaphysical account of properties is not forthcoming.

In order to see this, it will help to consider the array of metaphysical questions left unanswered by this functionalist theory. Consider, for instance, the Ramsey-Lewis definition of 'is $100^{\circ} \mathrm{C}$.' This does not provide an ontological analysis of the property. There is no guarantee that the functional predicate serves to designate a property at all, since there may be no property, or no unique property, that occupies the $F_{1}$ role. But even if we assume that there is, we are no closer to understanding what properties, ontologically speaking, are. They could be universals,

10 This, of course, assumes that properties, if not themselves causes and effects, can at least make a contribution to the causal workings of the universe. If just inputs are required for a property's having a functional role, it might be the case that properties could be epiphenomenal effects. In which case, the properties would have to form part of the causal nexus, whilst not themselves making any causal contribution. Either way, however, a position such as Davidson's (1967) would be excluded. 
sets of tropes, sets of possible particulars, etc. The Ramsey-Lewis definition leaves the whole range of theories wide open.

Similarly, the Ramsey-Lewis definition does not offer any indication of what kind of property this T-term refers to. Functional realisers may, of course, decide to specify the domain over which the existential quantifier in the definition ranges. For instance, they may decide to restrict it to scientifically respectable properties. Then, granted that they are correct, we can say that the predicate in question refers to one of these scientific properties. But the Ramsey-Lewis definition does not give us any such information. We only get this metaphysical claim out of the technique by putting it in in the first place.

Despite these limitations, however, it may be thought that the theory at least supplies a criterion of identity for properties. Since, in order for a functional predicate to refer to a property, that property must occupy the functional role specified in its Ramsey-Lewis definition. So it looks like we get this criterion of identity for properties:

In all possible worlds, two property instances are instances of the same property iff they realise the same functional role. ${ }^{11}$

If the Ramsey-Lewis definitions do commit us to this criterion of identity, then this would be a significant commitment. For the definitions would thereby specify the essences of properties.

A quick look at Lewis's work in the philosophy of mind, however, demonstrates that a functional realiser need not endorse this transworld criterion of identity. Lewis argues that the Ramsey-Lewis definitions for mental predicates such as 'is in pain' are non-rigid designators, as different properties can be designated by these definitions in different possible worlds. ${ }^{12}$ So, unless additional assumptions are incorporated into the Ramsey-Lewis technique, it does not follow from the fact that a

11 The criterion of identity offered above has the form of a two level criterion of identity, because it does not tell us when $\mathrm{x}$ and $\mathrm{y}$ are identical. Rather, it states that the function $f$ of $x$ is identical to the function $f$ of $y$ iff there is an equivalence relation between the entities over which variables $x$ and $y$ range (see Williamson 1990). As such, it contrasts with this one level criterion of identity which might be offered in its place: In all possible worlds, property $\mathrm{x}$ is identical to property $\mathrm{y}$ iff $\mathrm{x}$ and $\mathrm{y}$ occupy the same functional role. I think that the criterion of identity in question should be taken to be of the second form not the first, because what the functional definitions focus on is what instantiated properties enable a particular to do. In other words, we are claiming that a property is instantiated by a particular object iff a certain complex functional formula (laid out in its Ramsey-Lewis definition) is true of that object. But the conclusions of this section do not rest on this claim.

12 See, for instance, Lewis (1994). There he writes 'It's contingent, and it can only be known a posteriori, which physical states occupy which causal roles...'pain' is not a rigid designator' (p.303-4). 
property's predicate has a functional definition, that the property picked out by this definition must always realise this functional role. In other possible worlds (perhaps with different laws of nature, but perhaps not...) the property picked out by the definition may make a different causal contribution, so its functional role is not part of its essence.

What of a more restricted form of the criterion of identity? Surely we can at least say that, in the actual world, instantiated properties will realise the functional roles specified by their Ramsey-Lewis definitions? Not if we again follow the lead of Lewis, since he argues that, in special cases, a mental property need not occupy the functional role specified by its Ramsey-Lewis definition. ${ }^{13} \mathrm{He}$ famously asks us to suppose that nearly all tests show that $\mathrm{C}$-fibres firing satisfies the functional role specified by the predicate 'is in pain' in humans. However, there exists a mad human who displays none of the normal behaviour we associate with pain, but who instantiates the property of C-fibres firing when inflicted with injuries which would cause pain in the rest of us. In this case, Lewis argues, we would still have grounds for ascribing the property of being in pain to this person, as he instantiates the property that typically occupies the causal role of pain.

Lewis's response here shows that a criterion of identity for properties needn't follow from the functional realiser's Ramsey-Lewis definitions. While we discover what pain is via the causal relations described in its definition, once we have picked out that property, it stands as an independent entity, detachable from the causal relations that identified it. The reason for this is because, in Lewis's hands, the Ramsey-Lewis definitions serve an ideological, not metaphysical, purpose. They show us how we can identify a certain, problematic subset of properties, with a domain of, what are taken to be, acceptable properties. But the initial functional characterisation given by the Ramsey-Lewis definitions is not intended to characterise the nature of the property. So the criterion of identity stated above does not offer a necessary or sufficient condition for property identity.

There are, however, other functional realiser accounts that forge a closer link between a property and its functional role. Kim, for instance, argues that in order for a creature to instantiate a mental property, such as pain, the animal must display the causal relations specified in its functional definition and instantiate the typical realiser of pain for this sort of system with these laws of nature. ${ }^{14}$ So, contra Lewis, even if someone does instantiate the typical realiser of pain, the property cannot be attributed to them if it does not give rise to the behaviour definitive of pain.

\footnotetext{
13 See Lewis (1980).

14 See Kim (1998).
} 
Despite this, however, Kim still follows Lewis in treating the properties as independent entities, detachable from the functional relations that identify them. Kim argues that the Ramsey-Lewis definitions can designate different properties in different systems. So as well as occupying the functional role which is definitive of 'is in pain,' a creature must also instantiate the property which typically realises that role for its sort of system. Suppose, then, that $\mathrm{C}$-fibres firing is the occupier of the pain role in systems like humans, and D-fibres firing the occupant of the role in systems like dogs. Unless we are content to say that the predicate 'is in pain' refers to a gruesomely gerrymandered, disjunctive property, we are forced to say that $\mathrm{C}$-fibres firing is a different property from $\mathrm{D}$-fibres firing. So we cannot claim that two property instances are instantiations of the same property, namely pain, iff they both realise the functional role specified by the functional definition for pain. For these won't be two instances of the same property at all-one will be an instance of the property of $\mathrm{C}$-fibres firing and the other an instance of the property of D-fibres firing.

Kim endorses this conclusion, as he warns that we should not think of the properties designated by the Ramsey-Lewis definitions as functional properties, but rather as properties with functional characterisations. ${ }^{15}$ But it is clear that, as in the case of Lewis, these functional characterisations tell us very little about the nature of their referents. All Kim's view commits us to is the claim that a functional predicate, such as 'is in pain,' can only be ascribed to properties that occupy the same functional role within a certain system. But this falls way short of even the very limited criterion of identity outlined above for properties. As well as the aforementioned possibility of different properties realising the same functional role in different systems, one property may realise different functional roles in different systems. For instance, $\mathrm{C}$-fibres firing may realise the functional role of being in pain in humans, and being hungry in dogs. This makes it clear that Kim does not think that these functional predicates accurately mirror the natures of the properties in the pre-existing domain quantified over by the Ramsey-sentences. As a result, the Ramsey-Lewis definitions fail to provide substantive metaphysical claims about the nature of the properties designated by the functional, mental predicates.

Is there any point in trying to extend the functional realisers' use of these Ramsey-Lewis definitions to all properties? No, because it is very unclear what purpose this would serve. Functional realisers use the Ramsey-Lewis definitions to show how the T-terms can be analysed in terms of the already understood O-terms. For instance, in the philosophy of mind, they are used to show how mental properties can be understood in terms of, and so identified with, uncontroversial physical properties. 
Consequently, if we try to make wholesale use of the Ramsey-Lewis definitions, we thereby undermine the functional realiser's strategy. For, ex hypothesi, we are not attempting to understand or analyse a controversial domain of properties, those specified by the T-terms, in terms of an uncontroversial domain of properties, those specified by the already understood O-terms, as all our terms become T-terms. So the point of the functional realiser's analysis is lost when it is applied to all properties, as it requires a domain of O-terms that the T-terms are defined in terms of.

This does not mean, however, that the Ramsey-Lewis technique is incapable of performing a useful function within a theory of properties. Suppose, for instance, that one simply stipulates that properties are universals-perhaps because one has an independent argument for this view. ${ }^{16}$ Then, proponents of this variation of Shoemaker's causal theory of properties could use the technique to defend themselves against circularity, since it allows us to define several items simultaneously. So we can suppose that properties' predicates get analysed all at once via their causal roles. But this poses no threat to the modest claim I am making, for I fully accept that the Ramsey-Lewis technique can serve as a useful supplement to the causal theory of properties. My claim here is just that, on its own, this technique fails to provide the illuminating metaphysical analysis hoped for, since the Ramsey-Lewis definitions tell us nothing about the referents of the functional predicates. Consequently, extending the Ramsey-Lewis form of functionalism to all properties cannot help us to progress on the age-old question of what properties are.

In addition, we can also draw a more general conclusion, namely that it makes little sense to try to model a functional theory of properties upon the functional realiser's use of the Ramsey-Lewis technique. For if we generalise the analysis to cover all predicates, we don't have two sets of predicates - those specified by the controversial T-terms and those by the uncontroversial O-terms--which we are arguing refer to the same kind of (non-problematic) entity.

\section{IV}

Functional role theory is a very different kettle of fish from functional realiser theory. From the exposition given above, it should be clear that functional role theory does make metaphysical claims about the properties it analyses. For, on this view, functional properties are just functional roles, abstracted from their concrete realisations. Their whole

Following Armstrong (1997), for instance, it might be argued that the laws of nature have to be construed as second-order relations between universals, or that universals are the only entities capable of solving the One-Over Many problem. 
nature is exhausted by facts about what other entities will do, given certain circumstances.

This conception, not surprisingly, leads to some substantive metaphysical claims about the nature of functional properties. These properties have their functional roles essentially, as a functional property can only be instantiated at a world if there is something that realises the functional role definitive of it. Furthermore, the identity conditions for these properties are given in terms of their functional roles. If the functional role definitive of a property is realised, then this is both necessary and sufficient for that property to be instantiated. So we get the following criterion of identity: In all possible worlds, $\mathrm{x}$ and $\mathrm{y}$ are instantiations of the same property iff they realise the same functional role.

If we make wholesale use of this functional role analysis, therefore, and claim that all properties are functional role properties, we are going to be left with a substantive metaphysical theory of properties. This will not only specify the essential nature and identity conditions of a property, it also promises to capture Shoemaker's intriguing claim that 'properties are causal powers' (1980, p.210).

Trouble, however, is on the horizon. For while it makes sense to suppose that a certain subset of properties, like mental properties, are functional role properties, it is far from clear that the same is true when we extend the functional role analysis to all properties. The worry is that if we generalise functional role theory, and so say that all properties are functional role properties, then we're claiming that all properties consist in the having of some other property which occupies such-and-such a functional role. But of course if all properties consist in the having of some other properties which occupy such-and-such functional roles, there won't be any properties left to stand in such-and-such functional roles. Put another way, if there aren't any properties to be the concrete realisations of a functional role property's theory, their abstraction principle won't apply to anything. So no functional role properties will be abstracted from their concrete realisations.

Is this the death-blow to an extended form of functional role theory? Not quite. The standard characterisation of a functional role property allows for the possibility of their being realised by further functional role properties. Equally, nothing has been said to exclude functional role properties being the concrete realisations of other functional role properties. So the present difficulty is circumvented if there are always further functional role properties to realise the causal roles of distinct functional role properties.

This response, however, comes at a significant cost. For while the existence of properties does not strictly depend upon a domain of non-functional role properties, without them an infinite number of 
functional role properties have to be postulated. Any less than this, and there wouldn't always be a functional role property to realise suchand-such a functional role, so there wouldn't be any properties at all.

Could this commitment be avoided if functional role properties were the realisers of functional role properties that they themselves were realised by? So, crudely put, it would be possible for A to realise B, B to realise $\mathrm{C}, \mathrm{C}$ to realise $\mathrm{D} \ldots \mathrm{N}$ to realise $\mathrm{A}$. Yes, this would relieve us of the commitment, but by so doing, we would lose what is distinctive about functional role theory. To avoid functional role theory collapsing into functional realiser theory, strict constraints have to be imposed on the notion of realisation. In the abstraction principle, the concrete realisations of a property's theory must be taken to range over properties of an order lower than the functional role property in question. If this restriction isn't met, the specified property will be among the entities quantified over. So while the predicate which picks out the property will be of a higher-order, the property itself won't be. Consequently, the property in question won't be a functional role property, as it cannot be abstracted from its realisers.

In order to preserve the functional role analysis, therefore, properties must not be realised by properties of an order higher than themselves. So we can't avoid an infinite number of properties by making them realisers of themselves. Perhaps, then, we could just learn to live with them?

The difficulty with this lies not so much in the number of properties postulated, but rather in the nature of the commitment to this number of properties. Since we are interested in those properties which can partake in the causal nexus, the issue of how many properties there are seems to be an empirical one. If our scientific theories inform us that there are an infinite number of properties, fine. But to decide that matter a priori on the basis of a certain metaphysical theory of properties seems highly dubious. This is especially so when we remember that the view isn't just committed to an infinite number of properties, it is committed to an infinite number of instantiated properties. For the functional property of, for instance, being $100^{\circ} \mathrm{C}$ cannot be instantiated unless there are an infinite number of instantiations of other functional properties. So there have to be functional properties which are realisations of the theory for $100^{\circ} \mathrm{C}$, then further functional properties which are realisations of those properties' theories and so on ad infinitum.

What went wrong? Why does extending the functional role analysis to all properties lead to such unpalatable consequences? The answer, as with the functional realiser case, is that by making wholesale use of the functional role analysis, the very point of that analysis is lost. In order to see this, it will help to think about the functional role theorist's strategy in the philosophy of mind. Due to the perceived pressure of multiple-realisability, 
functional role theorists deny that mental properties can be identified with their realisers. But the physicalist leanings of these theorists mean that they still want to preserve the claim that all entities are, in some sense, physical. In order to capture both these claims, functional role theorists argue that mental properties are new, higher-order properties, which cannot be identified with their physical realisers. However, these properties do not require a non-physicalist substance or ontologically independent laws, because they are abstracted out of the physical realisations of their functional roles. This is what gives functional role theory its distinctive character. The particular functional role, specified by the property's theory, exhausts the nature of that property because functional role properties are viewed as logical constructions out of their physical realisers. So in order to formulate an analysis with the distinguishing commitments of functional role theory, it must parallel the constructive character of functional role accounts in the philosophy of mind.

This is why making wholesale use of the functional role analysis falls down. If we extend functional role theory to all properties, we cannot capture the constructive character of that analysis. For this requires a range of properties which are not subject to the functional role analysis. Without such a range of properties, we cannot regard functional role properties as logical constructions out of their concrete realisers. Although we can forever keep descending the hierarchy of more and more functional role properties, ex hypothesi, we never reach a level of properties which are not functional role properties. So a functional role analysis of all properties perpetually eludes us, since we never get to a range of entities in terms of which functional role properties can be understood. ${ }^{17}$

Such wholesale usage also conflicts with Russell's ramified theory of types, since if ail the predicates that specify properties are given Ramsey-Lewis definitions, all the predicates become of an order second or above, as they are all defined by the fact that some other property occupies such-and-such a functional role. As a result, the predicate quantifiers in the Ramsey-Lewis definitions of the second-order predicates have nothing to range over. For, according to the restrictions laid down by the ramified theory of types, the definition of a second-order predicate cannot quantify over entities picked out by second-order predicates. The account's failure to meet the constraints imposed by Russell's theory raises wider issues regarding the legitimacy of the use of this notion of a hierarchy. For it is not clear how the assumption that there is any such ordering of properties is justified, once we have postulated an infinite hierarchy of properties. This is because an infinite hierarchy of properties will not be well-founded, as there won't be a distinct base of fundamental properties, which higher-orders of properties in the hierarchy can be constructed from. In the absence of this, it is difficult to see how we can defend the claim that properties form a hierarchy, rather than just a collection, all of which are analysed in terms of one another. So, if this objection is not rebutted, the resulting functional role analysis will collapse into a form of functional realiser theory. 
It is important to grasp the nature of the problem here. The worry isn't that functional role properties can't be constructed out of other functional role properties, as I have already admitted that this is a possibility. Suppose, for instance, that we are after an understanding of mental properties and conclude that they are functional role properties, abstracted from neuropsychological properties. Given what has been said, it is perfectly consistent to then go on to say that these neuropsychological properties are themselves functional role properties, abstracted perhaps from biochemical properties. The present problem only arises because what we're after is a metaphysical analysis of all properties. As this is the aim, an endless series of functional role properties isn't going to be of any real assistance. Although such a procedure would provide us with analyses of kinds of properties - mental properties, neuropsychological properties and so on ad infinitum-it will not result in a metaphysical analysis of the category property. For in order to achieve a functional role analysis of all properties, we need to show how the type of entity in question, namely the properties, can be analysed as abstractions from a different kind of entity. Therefore, even with an infinite regress of functional role properties in place, still, we're unable successfully to implement the functional role theorist's strategy.

The hopes for a functionalist theory of properties seem to be fading fast. While we've found that the Ramsey-Lewis technique could be used as a supplement to a causal theory of properties, on its own it does not produce any informative metaphysical claims about the nature of properties. The position of an extended form of functional role theory appears even worse. Although it seemed to offer an ambitious metaphysical analysis of properties, on closer inspection the view looks completely unsustainable. The story doesn't end here, however. In the next section, I intend to revive the fortunes of a functionalist theory of properties, by proposing a functional role analysis which bypasses the problems outlined.

\section{V}

We've seen that the prospects for a generalised form of functional role theory look dire. For in order to preserve what is distinctive about this view, we're forced to postulate a number of properties that aren't analysed via functional role theory, thus abandoning the idea of extending this theory to all properties.

Is there any other way out? A possible alternative reveals itself when we notice that the term 'property' slides between two usages. Sometimes talk of properties refers to universals. These are unifying 
entities that can be instantiated in different particulars at the same time. It is, for instance, the universal of redness or roundness that makes different particulars alike in certain respects. In other contexts, the term 'property' is used to talk about instantiations of properties or property instances. Used in this way, the term does not refer to a universal which can be instantiated in different particulars at the same time, rather it picks out a single instantiation of that universal. These instances are inextricably tied to the particulars that instantiate them. A property instance is that cable's weakness or that ball's redness.

Regardless of what stance we adopt towards the ontology of properties and their instances, attention needs to be paid to this distinction between two senses of the term 'property,' as they name different kinds of entity. ${ }^{18}$ Take, for instance, two chocolate cakes which are both the same shade of brown. These cakes are alike in that they each instantiate the property of being that particular shade of brown, but is the colour of one cake identical to that of the other? If we think of properties in the first way, then the answer is yes, because they both share the same universal (whether universals are understood realistically or are reduced to something else). If, on the other hand, we think of properties in the second way, then the answer is no, because we have two instantiations of the universal of this shade of brown and, hence, two property instances of it.

This distinction between properties and their instances gives us more room to manoeuvre, as it draws our attention to a possible range of entities which could realise the functional roles of properties. Properties qua universals can be thought of as the functional role properties, while instances of these properties can be the entities which realise their functional roles.

More needs to be said, however, if this analysis is going to capture the constructive character of functional role theory. The concrete realisations of these functional role properties must be wholly distinct from the functional role properties they realise. Otherwise, the theory will be useless, as properties will be analysed as logical constructions out of a collection of entities which includes the very entities we are trying to analyse, namely, properties. This seriously curtails the theory that can be given of property instances. They cannot, for instance, be analysed as instantiations of universals, where universals are understood as sui

18 In what follows, I shall use the term 'universal' or 'property' to refer to the first sense of property, and the term 'property instance' or just 'instance' for the second. I do not suppose anything further by these terms. In particular, realism about universais is not implied, nor is realism about tropes. Property instances are meant to be neutral between instantiations of universals, tropes, members of sets, etc. 
generis entities, as in the accounts of Armstrong or Tooley. ${ }^{19}$ For, on these views, property instances are complexes of particulars and properties. So it would be circular to then attempt to construct properties out of property instances.

Another position that is excluded is the view that property instances are members of sets of possible particulars. On this analysis, property instances cannot be thought of as entities which are distinct from the sets of possible particulars of which they are part. This would make property instances far too coarse-grained, as the members of these sets are possible particulars, and possible particulars clearly instantiate numerous properties. So in order for these entities to count as property instances and not just particulars, they have to be thought of as part of a particular set or property. This means that functional role properties cannot be abstracted from a domain of property instances, since these instances are not metaphysically distinct from the properties or sets they are instances or members of.

The only analysis of property instances which views them as selfstanding entities, independent of the properties of which they are instances, is the view that property instances are sui generis entities or tropes. ${ }^{20}$ Tropes are very fine-grained entities which, while being property instances, are not analysed in terms of properties or partly constituted out of them. Indeed, most trope theorists believe that tropes are ontologically more basic than properties, since properties are often claimed to be sets of tropes. So it looks like these tropes could be the realisers we're looking for.

How, then, would the proposal go? If tropes are the realisers of a property's functional role, there will be many concrete realisations of its theory. If, for instance, this knife's sharpness realises a particular functional role, the sharpness of this qualitatively indistinguishable knife will also be able to. The multiple-realisability of these functional roles again undermines the uniqueness required for the Ramsey-Lewis definitions to work. But this can be regained by making the same move as functional role theorists in the philosophy of mind. So, by taking all the concrete realisations of a particular property's theory, we can abstract what is common to all of them by considering the

See Armstrong (1997) and Tooley (1987). Armstrong follows Aristotle in claiming that universals are immanent in their particulars, whereas Tooley, like Plato, thinks of them as outside space and time. But these differences do not matter for our purposes here, as both think of property instances as instantiations of these sui generis universals.

See, for instance, Williams (1953) and Campbell (1990). Although somewhat controversial, many of Husserl's followers take his moments to be sui generis property instances (see, for instance, Simons 1987). So these too would count as tropes, given their characterisation above. 
equivalence class of concrete realisations under the relation, sameness of functional role. In other words, we can say that two tropes are instances of the same property iff they both realise the same functional role. ${ }^{21}$ The functional role property can then be identified with this isolated property.

It may be objected, however, that the analysis is circular. If we say that two tropes are instances of the same universal because they realise the same functional role, then aren't we making an illicit appeal to properties or universals? For surely having the same functional role is a matter of having some property or universal. So the analysis of what it is to be an instance of a certain universal $F$ is just making an appeal to another universal: the universal of having the same functional role.

I don't think that this objection is successful, however, given a proper understanding of the theory. On the view being proposed, to say that two tropes realise the same functional role is not to claim that the two tropes instantiate some higher-order universal, that of having the same functional role. Rather it is to claim that these tropes make a certain functional role true of a particular. So, for instance, a particular instantiates the property of being $100^{\circ} \mathrm{C}$ iff it would burn human skin on contact, boil if water, etc. and a trope is what makes these conditionals true of that particular. The key claim is that if a certain functional formula is true of two particulars, then they instantiate the same property. And whilst this analysis, like other trope theories, takes for granted the notion of resemblance, since we have to grasp the idea that two particulars behave in similar ways in similar circumstances, the criterion of identity does not make an illicit appeal to irreducible universals or properties that tropes have in common.

But, one may protest, a particular could instantiate $F$ even if that particular never manifested any part of the functional role which made it $F$, since the circumstances never arise which render its F-ness apparent. Even if some of F's functional role is made manifest, in most

21 I do not wish to rule out the claim that tropes of different properties could realise the same functional role, so long as this is understood in a particular way. For, in cases of determinables and their determinates, I want to claim that both a trope of scarlet and a trope of crimson could realise the functional role of the determinable red (and thereby both be instances of red). But this is because these two determinate properties have distinct but overlapping functional roles, and the functional role of the determinable red is identified with this overlap. What is excluded is the claim that two tropes of different 'super-determinate' properties (i.e,, properties that are determinables of no other properties) could realise the same functional roles. If they did then, on this view, they would be instances of the same property. Of course, this is a very controversial claim that cannot be argued for here. However, those considerations that favour the more general causal theory of properties, will also support this thesis. 
instances, the particular will only exemplify a small fraction of the functional role definitive of $F$. Given this, although we can say that the particular is $\mathrm{F}$ iff it satisfies the functional role definitive of $\mathrm{F}$, we cannot say that how the object actually behaves is what makes it F. So what, then, are the truthmakers of the sentence, ' $a$ satisfies the functional role of $F$ '? What it is about the universe in virtue of which this sentence is true?

Appealing to the universal of F-ness at this point would, of course, just render the analysis circular, for in order to specify the functional role a trope has to realise to be an instance of $F$, we would have to invoke the very entity whose nature we are attempting to construct. However, just saying that a trope of F-ness realises such-and-such a functional role, as its particular would $\mathrm{X}$ in circumstances $\mathrm{C}_{1}, \mathrm{Y}$ in circumstances $\mathrm{C}_{2}$, etc., merely restates the problem. For if those circumstances are never actualised in this world, what is it that makes it the case that these counterfactuals are true of the object? Why is it that this trope would have enabled the object to $\mathrm{X}$, if it had been in circumstances $\mathrm{C}_{1}$ ? $^{22}$

At this point, the functionalist theory of properties has to dig its heels in. According to the view being proposed, tropes are the entities which bestow causal powers onto the world. They do not do this only when amalgamated with laws (be they Humean or non-Humean)-they are themselves dynamic entities that are the truthmakers of laws. So when we ask: 'Why is it that this trope would have enabled the object to $\mathrm{X}$, if it had been in circumstances $\mathrm{C}_{1}$ ?' all we can say is that this is because this is what F-tropes do. Tropes are the ground of irreducible power in the world, and it is because certain tropes bestow certain functional roles on particulars that they are instances of one property rather than another.

No doubt many will find this response inadequate, but now the battle needs to be fought on wider ground. Broadly speaking, there are three different approaches with respect to powers (and related causal

22

A slightly different, epistemological question the functionalist theory of properties raises is this: how do we know which property a trope is an instance of given that what it enables its particular to do can remain forever unmanifested? We discover what type of trope a particular instantiates by looking to see what the particular does. But there is always the possibility that had slightly different circumstances manifested themselves, we would have realised that we have tropes of different types on our hands. Whilst such possibilities cannot be excluded (and thus, unlike Shoemaker, I don't think that this analysis should be argued for on epistemological grounds) nevertheless, the view has some marked epistemological advantages over those which divorce the identity conditions for a property from what it does. (I argue for this claim in Whittle 2006. See Robinson 1994 for more details on these property permutation cases.) I thus think it would be short-sighted to reject the theory because of these epistemological worries. 
notions). The first, which this functionalist theory is an instance of, is the aforementioned causal theory of properties. ${ }^{23}$ This states that irreducibly dynamic properties (or, in this case, tropes) should be added into our ontology, since then we can offer better analyses of causal powers, causality, the laws of nature and arguably modality. ${ }^{24}$ Adherents of this approach thus pit themselves against both reductive, Humean analyses of powers and the non-Humean view that powers can be reduced to categorical properties and sui generis relations of nomic necessitation. Since the question of which stance is preferable is such an extensive one, it cannot be explored here. But, for our present purposes, what is important to stress is that, given this first view, we do have truthmakers for the causal counterfactuals we attribute to particulars. For the truthmaker for the proposition, 'if this particular had been in circumstances $C_{1}$, then it would have X-ed,' is just the actual, intrinsic persisting F-trope and the particular. Their existence alone entails the proposition.

The functionalist theory of properties, therefore, states that properties or universals are logical constructions out of tropes. The universal of F-ness, for instance, is abstracted from the collection of tropes which realise the functional role specified in F-ness' functional definition. This proposal still leaves issues outstanding. ${ }^{25}$ In particular, there is the important question of how this abstraction principle should be read. Until this is answered, the issue of what exactly it means to say that universals are logical constructions out of tropes is left undetermined. There are two options open to us here. Either we could embrace the reductionist reading, and identify universals with sets of tropes under the relation, sameness of functional role, or we could treat the universal as something distinct from the set of tropes, as functional role theorists do, but abstracted from them.

We saw that in the philosophy of mind, functional role theorists opt for the latter alternative, because they don't want to claim that mental properties are mere disjunctions of distinct physical properties. But there seems no analogous problem here, for although different tropes

23 This view is also often referred to as 'dispositional essentialism' (see, for instance, Ellis 2001).

24 See, for instance, Ellis (2001) and Molnar (2003).

25 One problem for this view, for instance, is that the account of universals offered entails that there are no uninstantiated universals. This causes difficulties once it is combined with the view that laws are relations between universals, since it appears to be logically possible for there to be basic laws of nature that, by accident, never have any instances. This, however, raises deep issues which cannot be addressed here. 
realise the functional roles of properties, they are all tropes of the same property. The question of whether an anti-reductionist or reductionist reading should be preferred, therefore, should turn upon whether there are any strong reasons for preferring a richer ontology of tropes and sui generis properties. If there aren't then, in the interests of parsimony, properties should be identified with sets of tropes under the relation, sameness of functional role.

Leaving this optional difference aside, however, the parallel between this unrestricted form of functionalism and functional role theory within the philosophy of mind is plain to see. In the philosophy of mind, functional role theorists postulate first-order physical properties, of which mental properties are taken to be logical constructions. A mental property, on this view, is instantiated just in case there is some physical property occupying the functional role specified by that mental property's functional theory. Likewise, here we find that tropes are analogous to the physical properties, as they are not logical constructions out of any other entities. Universals, by contrast, are comparable to mental properties, as they are constructed out of the tropes that realise their functional roles.

If we are prepared to endorse an ontology of tropes, therefore, an infinite hierarchy of properties is not required. Moreover, the functional role theorist's strategy can be implemented. For tropes can be the entities which realise the functional roles of properties, while properties can be abstracted from sets of tropes, all of which realise the same functional roles.

\section{VI}

The resulting functionalist theory of properties is extremely contentious. But this stems from a virtue of the account, namely that, unlike functional realiser theory, it does offer an informative analysis of properties. First, we are given an ontological analysis of properties, as properties are identified with sets of tropes which meet certain conditions specified in their Ramsey-Lewis definitions. ${ }^{26}$

Second, the theory results in this two level criterion of identity: In all possible worlds, two tropes are instances of the same property iff they both realise the functional role specified in that property's Ramsey-Lewis definition. The essential nature of a property is thus specified possibility would be to adopt the non-reductionist reading of the abstraction principle, but I shall leave this reading aside here. 
in terms of what its instances enable their particulars to do. As such, the analysis falls squarely within the tradition of causal theories of properties or dispositional essentialism. ${ }^{27}$ So the fate of the functional role analysis is very much tied to the success or failure of this approach. $^{28}$

Third, the analysis aids the cause of trope theory, by presenting us with a conception of what resemblance between tropes involves. The functional analysis offers a way of grouping tropes into sets which stand for natural properties. The set of tropes which is the property F-ness, on this view, consists of all those tropes which realise the functional role specified in the Ramsey-Lewis definition for property F-ness.

It may be objected that this is no advancement on the standard trope theorists' claim that properties are sets of tropes all of which resemble each other, since the present analysis fails to rid us of the notion of resemblance. I accept that the proposal still employs the notion of resemblance, since it appeals to the idea that particulars behave in similar ways in similar circumstances. But I nevertheless think that some progress is made. Tropes can be said to resemble each other in many ways. The tropes \{red trope, wise trope, charm trope\}, for instance, all resemble each other in some respect, since they are all members of this set. Trope theorists tend to exclude such abundant properties by appealing to the relation of 'precise similarity'. ${ }^{29}$ But this is problematic, because certain dissimilarities between tropes just aren't thought to matter. For instance, the fact that the tropes \{the scarlet trope of my left boot, the round trope of my blue ball $\}$ form a set, a set which no other scarlet trope is a member of, is not thought to show that the scarlet trope of my right boot is an instance of a different property from the scarlet trope of my left boot. What we need, then, is a better understanding of what the relevant relation of resemblance between tropes amounts to. And this is what the present proposal offers.

The analysis is also useful, more generally, in helping to distinguish between genuine and non-genuine resemblances between particulars. As in the case of tropes, we need to differentiate between those sorts of resemblances which are shared by particulars merely in virtue of belonging to the same set, and deeper resemblances such as having the

27 Whilst there are different formulations of this approach, all of its proponents claim that the causal contributions a property makes to a particular are essential features of that property. In addition to this, the issue regarding truthmakers raised earlier also places the functionalist theory of properties firmly within this camp. 
same mass, or the same charge ${ }^{30}$ Whilst the current proposal offers no reductive analysis of resemblance, it nevertheless makes the positive claim that tropes are the source of genuine resemblances between particulars. Any particular that satisfies the causal requirements laid down by a property's functional definition has a trope of that property. It is these tropes which realise the causal roles definitive of properties, that are the entities which ground genuine resemblances between particulars.

Finally, the proposal offers more content to Shoemaker's intriguing but elusive claim that 'properties are causal powers' (1980, p.210). On this view, it is true to say that properties are, in Armstrong's words, 'congealed hypothetical facts or states of affairs' (1997 p.79). For a property is instantiated by a particular just in case a certain cluster of causal conditionals (such as, if this water was heated to $100^{\circ} \mathrm{C}$, then it will boil...) is true of it. But this doesn't mean that the analysis runs 'counter to a very deep rooted way of thinking,' one which denies that when two particulars 'differ in their behaviour' there is 'some actual not merely hypothetical difference between them to account for this' ${ }^{31}$ For although properties can be thought of as mere functional roles, exhausted by facts about what particulars would do, on the present proposal, there are still the tropes, actual intrinsic states of particulars, that are there making these complex functional formulas true. These tropes are what justify our continued ascription of powers to particulars when they are not being manifested, and so are responsible for the truth of the causal counterfactuals we ascribe to them. On this view, if two particulars differ in causal powers, and thus in their causal counterfactuals, their tropes will also differ. So there are actual persisting states of particulars that are responsible for the ways in which particulars do, and could, behave.

It is possible, then, to formulate a functional role analysis of all properties. In order to avert disaster, we need to supplement functional role theory with an ontology of tropes. But once this has been done, a coherent and informative theory of properties emerges. ${ }^{32}$

30 See Lewis (1983). There he argues that the notion of a natural property that grounds objective resemblances between particulars does crucial work.

31 The quotes are taken from Geach (1957, p.5). The thesis that ascriptions of causal conditionals must be made true by persisting, occurrent states of those particulars is endorsed by a large number of philosophers (see, for instance, Armstrong, 1997 chapter 5).

This paper was written during my Jacobsen Fellowship at the University of London. I am deeply indebted for all the help and support offered by members of that institution. In particular, I would like to express my gratitude to Mike Martin, for his very extensive help and encouragement. Many thanks also to Tim Crane, Mark Kalderon, Janice Thomas, Joel Smith and an anonymous referee for all their detailed comments. 


\section{References}

Armstrong, David. (1997) A World of States of Affairs. Cambridge: Cambridge University Press.

Block, Ned. (1978) 'Troubles with Functionalism.' Minnesota Studies in the Philosophy of Science, IX. ed. W. Savage. Minneapolis: University of Minnesota Press: 261-326.

(1980) 'What is Functionalism.' in Readings in Philosophy of Psychology, 1. ed. N. Block. London: Methuen: 171-184.

Campbell, Keith. (1990) Abstract Particulars. Oxford: Blackwell.

Davidson, Donald. (1967) 'Causal Relations.' reprinted in his Essays on Actions and Events. (1980) Oxford: Clarendon Press: 149-162.

Dummett, Michael. (1991) Frege: Philosophy of Mathematics. London: Duckworth.

Ellis, Brian. (2001) Scientific Essentialism. Cambridge: Cambridge University Press.

Geach, Peter. (1957) Mental Acts. London: Routledge.

Hawthorne, John. (2001) 'Causal Structuralism.' Philosophical Perspectives: Metaphysics, 15: 361-378.

Kim, Jaegwon. (1998) Mind in the Physical World. Massachusetts: The MIT Press.

Lewis, David. (1970) 'How to Define Theoretical Terms.' The Journal of Philosophy, 67: 427-46.

(1980) 'Mad Pain and Martian Pain.' in his Philosophical Papers, I. (1983) Oxford: Oxford University Press: 122-132.

- (1983) 'New Work for a Theory of Universals.' reprinted in his Papers in Metaphysics and Epistemology. (1999) Cambridge: Cambridge University Press: 8-55.

_- (1994) 'Reduction of Mind.' reprinted in his Papers in Metaphysics and Epistemology. (1999) Cambridge: Cambridge University Press: 291-324.

Molnar, George. (2003) Powers: A Study in Metaphysics. Oxford: Oxford University Press.

Putnam, Hilary. (1967) 'Psychological Predicates.' in Art, Mind and Religion. ed. W. Captain and D. Merrill. Pittsburgh: University Of Pittsburgh Press: 37-48.

Robinson, Denis. (1993) 'Epiphenomenalism, Laws and Properties,' Philosophical Studies 69: 1-34.

Rosenberg, Alexander. (1984) 'Mackie and Shoemaker on Dispositions and Properties,' Midwest Studies Philosophy 7: 77-91.

Shoemaker, Sydney. (1980) 'Causality and Properties.' reprinted in his Identity, Cause and Mind. (1984) Cambridge: Cambridge University Press: 206-232. 
(1981) 'Some Varieties of Functionalism.' reprinted in his Identity, Cause and Mind. (1984) Cambridge: Cambridge University Press: 261-286.

Simons, Peter. (1987) Parts: A Study in Ontology. Oxford: Clarendon Press.

Tooley, Michael. (1987) Causation: A Realist Approach. Oxford: Clarendon Press.

Whittle, Ann. (2006) 'On An Argument for Humility.' Philosophical Studies 130: 461-497.

Williams, Donald. (1953) 'On the Elements of Being.' reprinted in Properties. eds. D. Mellor and A. Oliver (1997) Oxford: Oxford University Press: 112-124.

Williamson, Timothy. (1990) Identity and Discrimination. Oxford: Blackwell.

Wright, Crispin. (1983) Frege's Conception of Numbers as Objects. Aberdeen: Aberdeen University Press. 